\title{
MATERNAL FOETAL INTERACTIONS AND GENE FREQUENCY CHANGES IN POPULATIONS OF MICE
}

\author{
PETER HULL \\ Biology Department, Strathclyde University
}

Received 21.vi.71

\section{INTRODUGTION}

IT has been observed that, under certain circumstances, offspring of genotype like their mother have a smaller probability of survival than offspring of unlike genotype: Palm (1970) gives a summary of cases of maternal foetal interactions of this type which could lead to histocompatibility antigen polymorphism. In matings of mice where segregation was taking place at the $a$ locus (Hull, 1964), it has been suggested that this "autoincompatibility " is an immunological phenomenon and is due to the effects of a histocompatibility locus, or loci, on the same chromosome, rather than those of the alleles at the $a$ locus itself (Clarke and Kirby, 1966). It is know that the $H-3$ locus is approximately ten cross-over units away from the $a$ locus (Snell, 1958; Snell and Bunker, 1964). It has been shown (Hull, 1969) that certain incompatibility effects are associated with that region of chromosome V including the $H-3$ and $H-13$ loci (Snell et al., 1967), and that the two strains in the cross between which the maternal foetal incompatibility was first noted, differed in this chromosome region (Hull, 1971). In order to obtain further information on the cause of this incompatibility, the changes in gene frequency in isolated small populations of mice, derived from the stock of mice in which the autoincompatibility was first seen, were followed from generation to generation, and compared with those expected if autoincompatibility existed, either at the agouti locus itself or at a closely linked locus.

\section{EXPERIMENTAL MATERIAL}

The populations of mice used for this test were derived from a cross of two inbred strains known as $\mathrm{C} 3 \mathrm{Hf} / \mathrm{HeHa}$ (wild type at the $a$ locus) and $\mathrm{Hg} / \mathrm{Hu}\left(a^{t}\right.$ and $a$ alleles segregating). From the $+a^{t}$ mice obtained from this cross three closed populations were set up, either directly from the offspring of these $F_{1}$ mice or after not more than two generations of $+a^{t} \times+a^{t}$ mating. In addition to this, two populations were set up later, using mice from the other lines, surplus to the requirements for their maintenance. The large cages used to contain the populations of mice were made up from eight standard $7^{\prime \prime} \times 11^{\prime \prime} \times 4^{\prime \prime}$ plastic mouse cages, each provided with food and water, connected by a system of enclosed wire mesh runs. It was hoped that this arrangement would allow the mice to come into contact with one another but allow the loser of any fight to avoid death or serious injury by escaping from the victor.

Every large cage was examined each day and pregnant females were removed to individual cages, where they were allowed to rear their young. The young mice were classified at weaning, when the three phenotypes: agouti $(++)$; agouti and $\tan \left(+a^{t}\right)$ and black and $\tan \left(a^{t} a^{t}\right)$, could be 
distinguished. Each mouse of the new generation was numbered by an ear clip before being weaned at the age of 3 weeks and placed in a new large cage. Any dead mice were removed immediately they were seen. The mice were left in the large cage until either all the females had become pregnant or until 6 weeks after the first female had produced a litter. Then at this stage all the mice remaining in the cage were discarded.

Frequently the number of mice in the offspring generation was from two to three times the number of mice which had been put in the cage as potential parents. To keep a fairly constant number of parents (at least 25), the mice were reduced in number by retaining only every second or every third mouse of each genotype of each sex, in order of age, in these generations. If fewer than 25 mice were available for any line, it was discontinued.

Changes in gene frequency were followed from generation to generation and compared with those expected in a population of the size of the particular generation. It was considered reasonable to calculate the relative genotypic frequencies among female parents from fertile females only, i.e. those which produced a viable litter and thus contributed to the next generation. There was no way of knowing which males mated. These facts will be considered when the results of the experiment are examined in detail. The experiment was terminated after nine generations in the two longest established lines as the facilities for keeping the mice were no longer available.

\section{ANalysis of Results}

Using a computer and given any set of male and female parents in any one generation, the expected gene frequency, with a particular type of selection, in the next generation may be calculated. It is also possible to calculate, knowing the number of offspring $(k)$ produced in this second generation, the probability of the production of all possible sets of offspring, e.g. a set of $\left[k a^{t} a^{t}\right]$, a set of $\left[(k-1) a^{t} a^{t} ; 1+a^{t}\right]$, etc., and from these we may then combine the probabilities of those sets of offspring, each of which would give rise to the same gene frequency of the + allele, $p_{b}$, in this second generation. From this frequency distribution of probabilities it is possible to calculate, for each generation, the total probability of a shift in gene frequency as extreme as, or more extreme than, that actually observed. Each generation of mating and selection is a replicate of every other, with the same selective forces, the only difference being in the array of parental genotypic frequencies. If the probability of the observed gene frequency shift in the $j$ th generation is transformed to $\chi_{j}, \quad \chi_{j}$ being given a positive value for a gene frequency of the $a^{t}$ allele greater than that expected and a negative value for a frequency less than that expected), then these $n$ values of $\chi_{j}$ may be summed, $n$ being the total number of generations of mating and selection in all five lines, and hence the probability of the total movement in gene frequency for the whole experiment may be obtained from $t_{n-1}=$ $\sum_{j=1}^{n} \chi_{j} / \sqrt{ } \sqrt{n}$. A very low total probability would then suggest that the assumptions made about the selective forces, mating behaviour or some other aspect of the experiment involved in calculating the expected gene frequency in each generation was invalid.

The analysis was performed three times; the overall probability of the observed gene frequency changes was calculated, assuming random mating: 
(a) with no selection;

(b) with autoincompatibility;

(c) with autoincompatibility at a locus 10 crossover units from the $a$ locus.

Taking specific examples, if among the parents:

$D_{f}=$ frequency of $++q$ mice; $\quad D_{m}=$ frequency of $++\delta$ mice;

$H_{f}=$ frequency of $+a^{t}$ \% mice; $\quad H_{m}=$ frequency of $+a^{t} \delta^{t}$ mice;

$R_{f}=$ frequency of $a^{t} a^{t}$ o mice; $\quad R_{m}=$ frequency of $a^{t} a^{t} \delta^{t}$ mice;

and $D_{f}+H_{f}+R_{f}=D_{m}+H_{m}+R_{m}=1$, then the expected gene frequency among the offspring may be calculated as follows:

In case (a), where there is no selection, the expected frequencies $D, H$, and $R$, of the three genotypes,$+++a^{t}$ and $a^{t} a^{t}$ among the offspring would be (table 1):

$$
\left.\begin{array}{l}
D=D_{f} D_{m}+\frac{1}{2}\left(D_{f} H_{m}+H_{f} D_{m}\right)+\frac{1}{4} H_{f} H_{m} \\
R=R_{f} R_{m}+\frac{1}{2}\left(H_{f} R_{m}+R_{f} H_{m}\right)+\frac{1}{4} H_{f} H_{m} \\
H=1-D-R
\end{array}\right\}
$$

TABLE 1

Frequencies of matings and offspring produced, with no selection

\section{Type of mating 우 8}

$++x++$

$++x+a^{t}$

$++\times a^{t} a^{t}$

$+a^{2} x++$

$+a^{t} x+a^{t}$

$+a^{t} \times a^{t} a^{t}$

$a^{t} a^{t} x++$

$a^{t} a^{t} \times+a^{t}$ $a^{t} a^{t} \times a^{t} a^{t}$
Type of offspring produced

$\overbrace{D}^{++}+\underset{H}{+a^{t}} \quad a_{R}^{t} a^{t}$

$D_{f} D_{m}$
$D_{f} H_{m}$
$D_{f} R_{m}$
$H_{f} D_{m}$
$H_{f} H_{m}$
$H_{f} R_{m}$
$R_{f} D_{m}$
$R_{f} H_{m}$
$R_{f} R_{m}$

$D_{f} D_{m}$

$\frac{1}{2} D_{f} H_{m}$

$1 H_{f} D_{m}$

$\frac{1}{1} H_{f} H_{m} \quad \frac{1}{2} H_{f} H_{m}$

$\frac{1}{2} H_{f} R_{m}$

$R_{f} D_{m}$

$\frac{1}{2} R_{f} H_{m}$

${ }_{1}^{1} H_{f} H_{m}$ $\frac{1}{2} H_{f} R_{m}$

$\frac{1}{2} R_{f} H_{m}$ $R_{f} R_{m}$

The frequency distribution of probabilities of the $2 k+1$ different gene frequencies of the + allele $p_{b}$, where $b$ runs from 0 to $2 k$, with $k$ offspring, will then be given by:

$$
1=\sum_{b=0}^{2 k} \sum_{\substack{i=b-k \\ i \neq 0}}^{b / 2} \frac{k !}{i !(b-2 i) !(k+i-b) !} D^{i} H^{b-2 i} R^{k+i-b}
$$

To speed the calculations it was more convenient to generate by the computer a table of $\ln x$ ! and to use the form:

$$
\begin{aligned}
1=\sum_{b=0}^{2 k} \sum_{\substack{i=b-k \\
i \neq 0}}^{b / 2} \exp (\ln k !+i \ln D+(b-2 i) \ln H \\
\quad+(k+i-b) \ln R-\ln i !-\ln (b-2 i) !-\ln (k+i-b) !)
\end{aligned}
$$

If we assume, in case (b) that autoincompatibility of the type previously shown to be associated with the $+/ a^{t}$ system (Hull, 1964) is directly due to the alleles at the $a$ locus i.e. that the probabilities of survival of:

$$
\left.\begin{array}{c}
++ \text { offspring from }++ \text { mother is } 1-s_{1} \\
+a^{t} \text { offspring from }+a^{t} \text { mother is } 1-s_{2} \\
a^{t} a^{t} \text { offspring from } a^{t} a^{t} \text { mother is } 1-s_{3}
\end{array}\right\}
$$


then we may replace equations (1) by the set (table 2):

$$
\left.\begin{array}{c}
D=D_{f} D_{m}+D_{f} H_{m} \frac{1-s_{1}}{2-s_{1}}+H_{f} D_{m} \frac{1}{2-s_{2}}+H_{f} H_{m} \frac{1}{4-2 s_{2}} \\
R=R_{f} R_{m}+R_{f} H_{m} \frac{1-s_{3}}{2-s_{3}}+H_{f} R_{m} \frac{1}{2-s_{2}}+H_{f} H_{m} \frac{1}{4-2 s_{2}} \\
H=1-D-R
\end{array}\right\}
$$

The values used were $s_{1}=-0.036, s_{2}=+0.191$ and $s_{3}=+0.307$ (Hull, 1964). From (3) the new $p_{b}$ vectors appropriate to this type of selection may again be generated.

TABLE 2

Frequencies of matings and offspring produced, with autoincompatibility

\begin{tabular}{|c|c|c|c|c|}
\hline \multirow{2}{*}{$\begin{array}{l}\text { Type of } \\
\text { mating }\end{array}$} & \multirow[b]{3}{*}{ Frequency } & \multicolumn{3}{|c|}{ Type of offspring produced } \\
\hline & & ++ & $+a^{t}$ & $a^{t} a^{t}$ \\
\hline 의 & & $D$ & $H$ & $R$ \\
\hline$++x+t$ & $D_{f} D_{m}$ & $D_{f} D_{m}$ & & \\
\hline$++x+a^{t}$ & $D_{f} H_{m}$ & $D_{f} H_{m} \frac{1-s_{1}}{2-s_{1}}$ & $D_{f} H_{m} \frac{1}{2-s_{1}}$ & \\
\hline$++\times a^{t} a^{t}$ & $D_{f} R_{m}$ & & $D_{f} R_{m}$ & \\
\hline$+a^{t} x++$ & $H_{f} D_{m}$ & $H_{f} D_{m} \frac{1}{2-s}$ & $H_{f} D_{m} \frac{1-s_{2}}{2-s}$ & \\
\hline$+a^{t} x+a^{t}$ & $H_{f} H_{m}$ & $H_{f} H_{m} \frac{1}{4-2 s}$ & $H_{f} H_{m} \frac{1-s_{2}^{2}}{2-s}$ & $H_{f} H_{m} \frac{1}{4-2 s}$ \\
\hline$+a^{t} \times a^{t} a^{t}$ & $H_{f} R_{m}$ & & $H_{f} R_{m} \frac{1-s_{2}^{2}}{2-s}$ & $H_{f} R_{m} \frac{1}{2}$ \\
\hline$a^{t} a^{t} x++$ & $R_{f} D_{m}$ & & $R_{f} D_{m}$ & \\
\hline$a^{t} a^{t}+\times a^{t}$ & $R_{f} H_{m}$ & & $R_{f} H_{m} \frac{1}{2-s_{3}}$ & $R_{f} H_{m} \frac{1-s_{a}}{2-s_{a}}$ \\
\hline$a^{t} a^{t} \times a^{t} a^{t}$ & $R_{f} R_{m}$ & & & $R_{f} R_{m}$ \\
\hline
\end{tabular}

In case (c), autoincompatibility due to a gene 10 cross-over units from the $a$ locus, it was necessary to reduce the autoincompatibility values (4) in each successive generation in order to simulate crossing over between the coat colour marker gene and the linked gene, supposedly responsible for the maternal foetal interaction, before substituting in (5) and (3). As not more than half of the chromosomes would be expected to be present in heterozygous condition in each generation of random mating, and the $a$ and $H-3$ loci are about ten cross-over units apart, it is considered reasonable as a first approximation to reduce the autoincompatibility values (4) to 95 per cent. of their previous value for every generation which elapsed between the original cross of the two inbred strains and the generation concerned.

\section{Results}

The frequency of the $a^{t}$ allele in each of the five lines is plotted against generation in fig. 1. The number of offspring from which the gene frequency was calculated in each generation for the five lines $\mathrm{A}$ to $\mathrm{E}$ is given in table 3. The unweighted regression of the frequency of the $a^{t}$ allele on generation, pooled for the five lines is $-0 \cdot 0079 \pm 0 \cdot 0056$. This calculation however takes no account of the number of offspring on which the gene frequencies were based. For example, the largest shifts in gene frequency occurred in generation 6 of lines $\mathrm{C}$ and $\mathrm{D}$, when the numbers of offspring were low-in fact fewer than 25 , which had previously been decided on as the criterion for discontinuing the lines. 


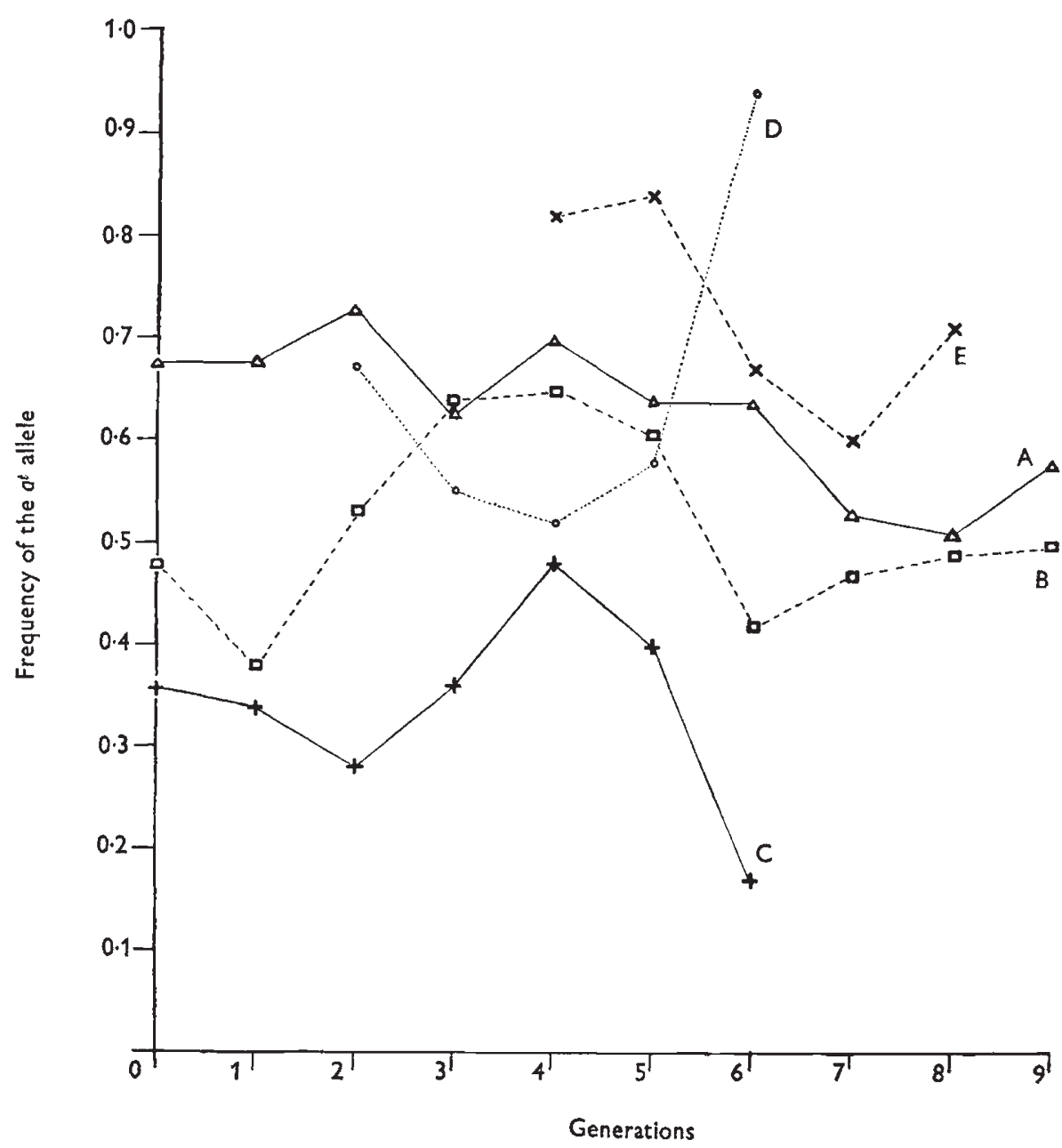

Frg. 1.-Frequency of the $a^{t}$ allele in each generation of the five populations.

In table 3 are given the total numbers of mice placed in each cage for the five lines for each generation, the total number of offspring produced from each cage and the gene frequency among these offspring. Then follow the $\chi$ values based on the probability of obtaining a shift in gene frequency as extreme as that observed on the basis of the three hypotheses: (a) that no selection was taking place; (b) that there was incompatibility at the $a$ locus; or (c) that there was incompatibility at a linked locus, ten cross-over units from the $a$ locus.

Two types of contribution to the change in gene frequency in each line are of interest : factors causing a consistent change, possibly due to continued operation of autoincompatibility selection, or a random component, due to the finite size of the population in each generation. The total value of $\chi$ for all lines, assuming no selection was $-14 \cdot 43$. Thus:

$$
t_{31}=-\left(32^{-\frac{1}{2}}, 14.43\right)=-2.55, \mathrm{P}<0.02,
$$


TABLE 3

Gene frequency and $\chi$ for the three populations

\begin{tabular}{|c|c|c|c|c|c|c|c|}
\hline \multirow[b]{2}{*}{ Line } & \multirow[b]{2}{*}{$\begin{array}{l}\text { Gene- } \\
\text { ration }\end{array}$} & \multirow[b]{2}{*}{$\begin{array}{c}\text { Number } \\
\text { of } \\
\text { mice }\end{array}$} & \multirow[b]{2}{*}{$\begin{array}{c}\text { Number } \\
\text { of } \\
\text { offspring }\end{array}$} & \multirow[b]{2}{*}{$\begin{array}{c}\text { Frequency } \\
\text { of } a^{t} \\
\text { allele }\end{array}$} & \multicolumn{3}{|c|}{$\chi$ for different types of selection } \\
\hline & & & & & $\begin{array}{c}\text { No } \\
\text { selection }\end{array}$ & $\begin{array}{l}\text { Incompatibility } \\
\text { at } a \text { locus }\end{array}$ & $\begin{array}{l}\text { Incompatibility } \\
\text { at linked locus }\end{array}$ \\
\hline \multirow{10}{*}{ A } & 0 & - & - & 0.67 & - & - & - \\
\hline & 1 & 36 & 61 & 0.68 & +0.10 & +0.29 & +0.29 \\
\hline & 2 & 61 & 47 & 0.73 & $+2 \cdot 25$ & +2.38 & $+2 \cdot 20$ \\
\hline & 3 & 27 & 97 & 0.63 & -1.31 & -1.29 & $-1 \cdot 22$ \\
\hline & 4 & 50 & 156 & 0.70 & +3.12 & +3.58 & +3.43 \\
\hline & 5 & 52 & 92 & 0.64 & $-2 \cdot 40$ & $-2 \cdot 20$ & $-2 \cdot 21$ \\
\hline & 6 & 49 & 45 & 0.64 & 0.00 & +0.12 & +0.11 \\
\hline & 7 & 45 & 86 & 0.53 & -3.97 & -3.78 & -3.83 \\
\hline & 8 & 45 & 48 & 0.51 & +0.02 & +0.31 & +0.31 \\
\hline & 9 & 48 & 50 & 0.58 & -0.93 & -0.72 & -0.72 \\
\hline \multirow{10}{*}{ B } & 0 & - & - & 0.48 & - & - & 一 \\
\hline & 1 & 32 & 56 & 0.38 & $-2 \cdot 19$ & $-2 \cdot 15$ & $-2 \cdot 15$ \\
\hline & 2 & 56 & 97 & 0.53 & $+2 \cdot 80$ & +3.35 & +3.35 \\
\hline & 3 & 52 & 63 & 0.64 & +1.28 & +1.03 & +1.04 \\
\hline & 4 & 63 & 182 & 0.65 & +0.15 & +0.49 & +0.38 \\
\hline & 5 & 45 & 59 & 0.61 & -0.48 & -0.47 & -0.48 \\
\hline & 6 & 32 & 43 & 0.42 & $-3 \cdot 46$ & $-3 \cdot 38$ & -3.43 \\
\hline & 7 & 43 & 66 & 0.47 & +0.09 & +0.25 & +0.26 \\
\hline & 8 & 66 & 52 & 0.49 & +0.30 & +0.48 & +0.49 \\
\hline & 9 & 52 & 15 & 0.50 & -0.55 & -0.19 & -0.17 \\
\hline \multirow{7}{*}{ C } & 0 & - & - & 0.36 & - & - & - \\
\hline & 1 & 36 & 67 & 0.34 & -0.28 & -0.09 & -0.09 \\
\hline & 2 & 35 & 36 & 0.28 & -1.36 & $-1 \cdot 10$ & $-1 \cdot 10$ \\
\hline & 3 & 36 & 80 & 0.36 & +0.94 & +1.28 & +1.28 \\
\hline & 4 & 42 & 50 & 0.48 & +0.51 & +0.71 & +0.71 \\
\hline & 5 & 50 & 25 & 0.40 & -0.99 & -0.96 & -0.97 \\
\hline & 6 & 25 & 21 & $0 \cdot 17$ & -3.05 & $-2 \cdot 68$ & -3.08 \\
\hline \multirow{5}{*}{ D } & 0 & - & - & 0.67 & - & - & - \\
\hline & 1 & 37 & 71 & 0.55 & $-3 \cdot 12$ & -2.93 & -2.94 \\
\hline & 2 & 37 & 89 & 0.52 & -0.38 & -0.37 & -0.37 \\
\hline & 3 & 47 & 46 & 0.58 & -0.12 & 0.00 & 0.00 \\
\hline & 4 & 25 & 17 & 0.94 & $+2 \cdot 59$ & +3.02 & $+2 \cdot 70$ \\
\hline \multirow{7}{*}{ E } & 0 & - & - & 0.82 & - & - & - \\
\hline & 1 & 36 & 62 & 0.84 & +0.36 & +0.35 & +0.36 \\
\hline & 2 & 31 & 36 & 0.67 & -2.75 & -2.52 & $-2 \cdot 60$ \\
\hline & 3 & 36 & 55 & 0.60 & -1.60 & -1.56 & -1.57 \\
\hline & 4 & 55 & 28 & 0.71 & 0.00 & +0.15 & +0.15 \\
\hline & & & & $\Sigma \chi$ & -14.43 & $-8 \cdot 60$ & -9.87 \\
\hline & & & & $\Sigma \chi^{2}$ & 106.02 & 107.34 & $107 \cdot 00$ \\
\hline
\end{tabular}

indicating that hypothesis (a), that selection is not operating, is invalid. If the hypothesis is that there is autoincompatibility at the $a$ locus we have:

$$
t_{31}=-\left(32^{-\frac{1}{2}} \cdot 8 \cdot 60\right)=-1 \cdot 42, \mathrm{P} \simeq 0 \cdot 16,
$$

in accordance with hypothesis (b). However, if the hypothesis is (c), that autoincompatibility is operating at a linked locus ten cross-over units away, then:

$$
t_{31}=-\left(32^{-\frac{1}{2}} \cdot 9 \cdot 87\right)=-1 \cdot 74, \mathrm{P} \simeq 0.09
$$


It must be concluded that it is not possible to distinguish by this method between the validity of hypotheses (b) and (c) from these data.

For all three cases the value of $\sum \chi_{31}^{2}$ is very highly significant. For any of the three hypotheses, the amount of random gene frequency fluctuation from generation to generation is greater than that expected. The expected gene frequency among offspring was calculated from the relative genotypic ratios of fertile females only. If, however, only a proportion of the males mated with the available females (which seems probable, since it is thought that each female would usually mate only once and those males which became sexually mature earliest would thus mate with most of the females), this would lead to greater fluctuations in gene frequency than that expected between generations. Nevertheless, as long as there was no correlation between genotype and fertility in the male, this would not lead to any systematic change in gene frequency of the type observed. Similarly, variation in litter size between females could also lead to random changes in gene frequency, if there is no correlation between litter size and genotype. The mean litter sizes at weaning, based on pooled data from all five lines for each of the three types of female were extremely similar: ++ o $=5 \cdot 623 ;+a^{t}$ 우

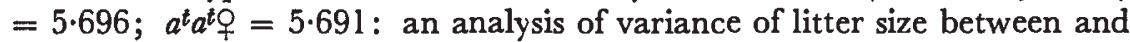
within genotypes for all five lines gave $F_{354}^{2}=0.04$. Thus there is no evidence of differences in fertility between females of the three genotypes as a possible cause of changes in gene frequency.

From these data it is possible to say that some selective force or forces are causing the slow replacement of the $a^{t}$ allele by the + allele at the rate of about 1 per cent. per generation. The hypothesis that this change is purely the result of chance is very unlikely to be true. The data are compatible with the hypotheses either that autoincompatibility of a degree observed previously occurs at the $a$ locus itself or at a locus ten cross-over units away. To distinguish between these two possibilities either a much larger experiment, i.e. with more separate populations would have been needed at this stage, when a high proportion of the chromosomes would still be expected to be in the same configuration in this region as when received from the inbred lines, or the populations would have had to be continued for a much longer time to allow alleles at the supposed two separate loci to approach linkage equilibrium.

\section{Summary}

1. Gene frequency changes were observed at the $a$ locus in five closed populations of laboratory mice.

2. There were very large random changes in gene frequency from generation to generation. Systematic changes also occurred, the $a^{t}$ allele being replaced by the + allele.

3. These changes were similar to those which would have been expected with autoincompatibility at the $a$ locus of a magnitude already reported. However, enough information could not be obtained from the data available by the method used, to distinguish between this possibility and that that the autoincompatibility was at a linked locus ten cross-over units from the $a$ locus.

Acknowledgments.-Part of this work was done during the tenure of a research fellowship at the University of Liverpool, Department of Genetics, where much help was given by

02 
Professor P. M. Sheppard. Discussion with Professor R. C. Lewontin is gratefully acknowledged. Assistance with the computations was given by Miss E. Russell.

\section{REFERENCES}

CLARKE, B., AND KIRBY, D. R. S. 1966. Maintenance of histocompatibility polymorphisms. Nature, 211, 999-1000.

HULL, P. 1964. Partial incompatibility not affecting litter size in the mouse. Genetics, 50, 563-570.

HULL, P. 1969. Maternal-foetal incompatibility associated with the $H-3$ locus in the mouse. Heredity, 24, 203-209.

HULL, P. 1971. The fifth chromosome histocompatibility types of mouse strains $\mathrm{Hg} / \mathrm{Hu}$ and C3Hf/A. Heredity, 26, 140-145.

PALM, J. 1970. Maternal fetal interactions and histocompatibility antigen polymorphisms. Transplantation Proceedings, 2, 162-173.

SNELL, G. D. 1958. Histocompatibility genes of mice. I. Demonstration of weak histocompatibility differences by immunisation and controlled tumor dosage. F. Nat. Cancer Inst., 20, 787-824.

SNELL, G. D., AND BUNKER, H. P. 1964. Histocompatibility genes of mice. IV. The position of $H-3$ in the fifth linkage group. Transplantation, 2, 743-751.

SNELL, G. D., CUDKOWICZ, G., AND BUNKER, H. P. 1967. Histocompatibility genes of mice. VII. $H$ - 13 , a new histocompatibility locus in the fifth linkage group. Transplantation, 5, 492-503. 